\title{
Solid Lipid Nanoparticles for image-guided therapy of arthrosclerosis
}

Khalid Oumzil, ${ }^{a, b}$ Michael A. Ramin, ${ }^{a, b}$ Cyril Lorenzato, ${ }^{c}$ Audrey Hémadou, ${ }^{c}$ Jeanny

Laroche, ${ }^{c}$ Marie Josée Jacobin-Valat ${ }^{c}$ Stephane Mornet, ${ }^{d}$ Claude-Eric Roy, ${ }^{a, b}$ Tina Kauss, ${ }^{a, b}$ Karen Gaudin,,$^{a, b}$ Gisèle Clofent-Sanchez ${ }^{c}$ and Philippe Barthélémy**a,b

${ }^{a}$ Univ. Bordeaux, ARNA laboratory, F-33000 Bordeaux, France.

bINSERM, U869, ARNA laboratory, F-33000 Bordeaux, France.

${ }^{\mathrm{c} C R M S B}$ Centre de Résonance Magnétique des Systèmes Biologiques, UMR 5536, CNRS, Univ. Bordeaux, F-33076 Bordeaux, France

${ }^{\mathrm{d}}$ Institut de Chimie de la Matière Condensée de Bordeaux, ICMCB UPR CNRS 9048, Univ.

Bordeaux, F-33608 Pessac, France

* corresponding author: philippe.barthelemy@inserm.fr

Supporting Information

A. Characterization of iron oxide nanoparticles clusters with nucleolipid ............... 2

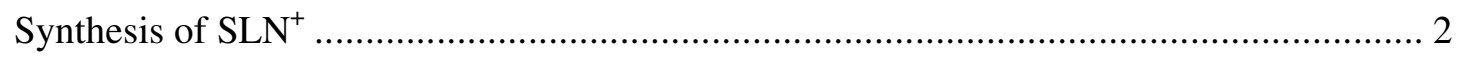

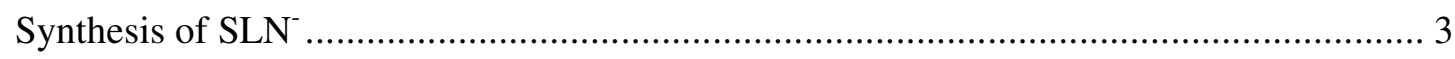

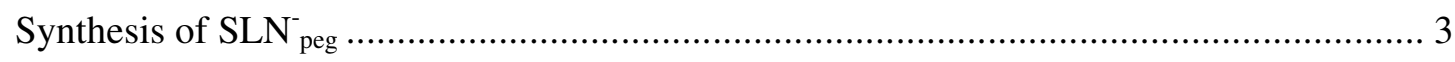

Control (DOPC with iron oxide nanoparticles) ........................................................... 4

B. Characterization of iron oxide nanoparticles clusters with nucleolipid and API... 5

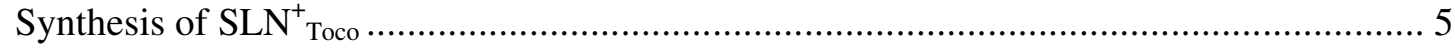

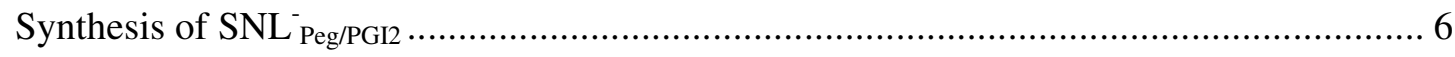

C. Preparation of samples for HPLC analysis and dosage of DOTAU and $\alpha$ tocopherol $\left(\mathrm{SNL}^{+}\right.$and $\mathrm{SNL}^{+}{ }_{\text {Toco }}$ ) 


\section{A. Characterization of iron oxide nanoparticles clusters with nucleolipids}

\section{Synthesis of SLN ${ }^{+}$}

Encapsulation of iron oxide nanoparticles clusters by DOTAU

Size Distribution by Intensity

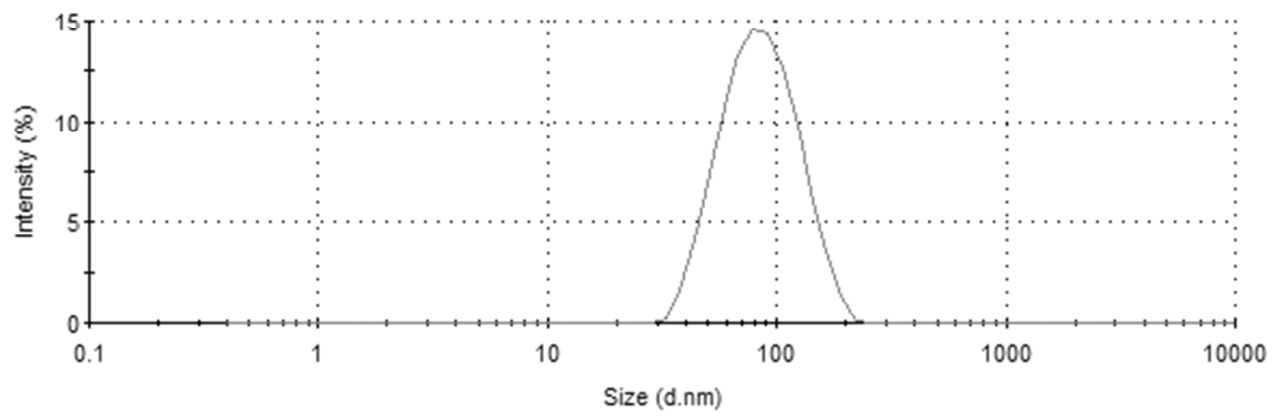

Figure SI1. Size distribution measurement of SLN ${ }^{+}$.

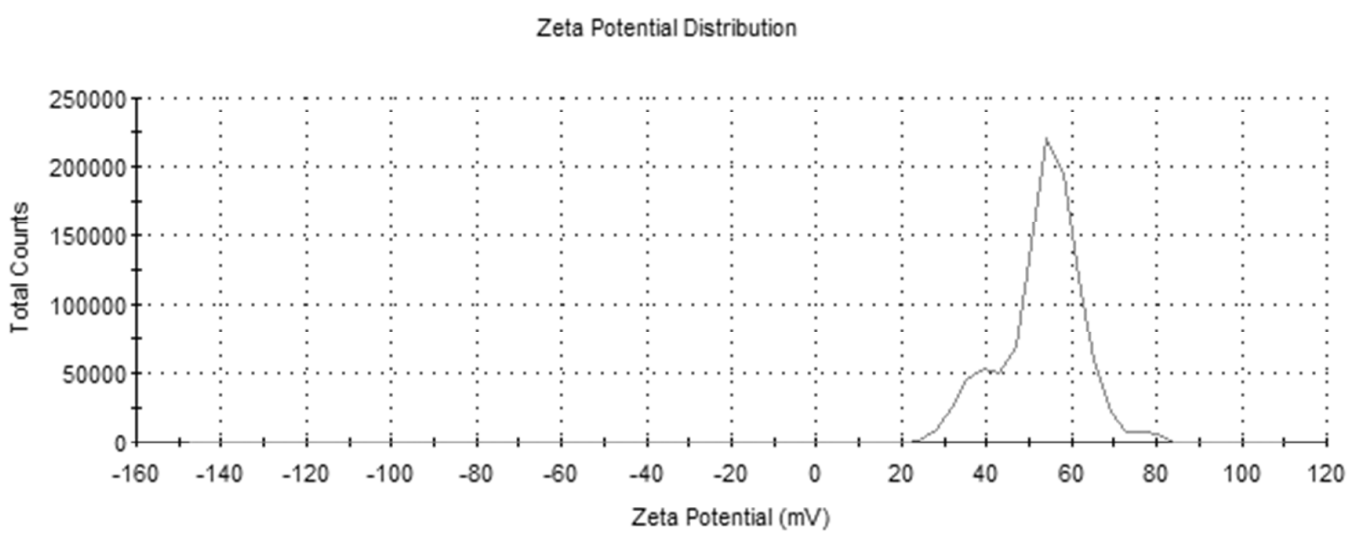

Figure SI2. Zeta potential distribution measurement of SLN ${ }^{+}$.

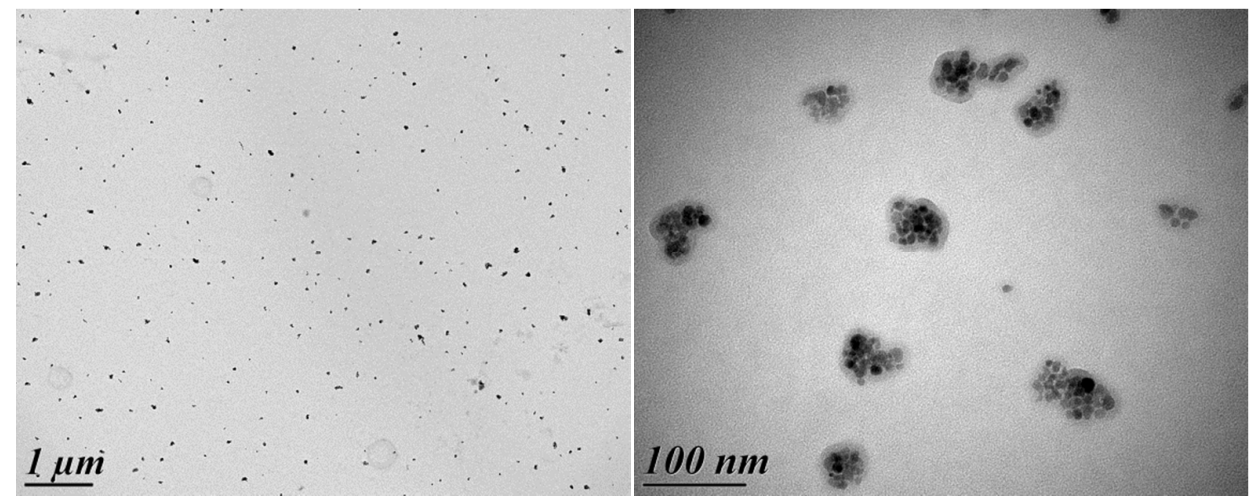

Figure SI3. TEM images showing the grapes of nanoparticles stabilized with DOTAU $\left(\mathrm{SLN}^{+}\right)$. 


\section{Synthesis of SLN}

Encapsulation of iron oxide nanoparticles clusters by diC16dT

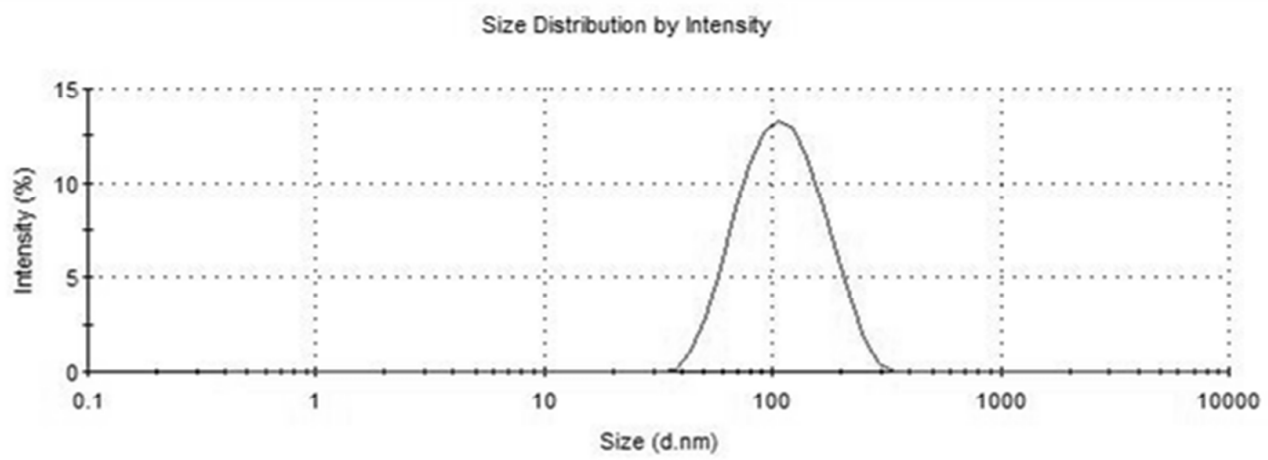

Figure SI4. Size distribution measurement of SLN ${ }^{-}$.

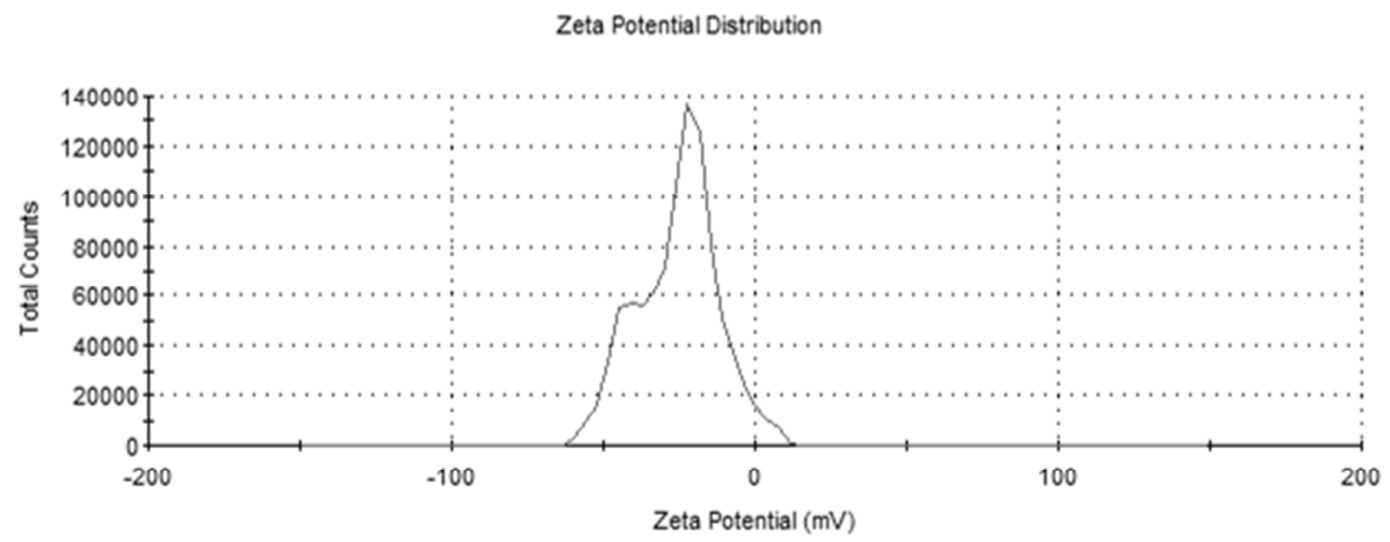

Figure SI5. Zeta potential distribution measurement of SLN ${ }^{-}$.

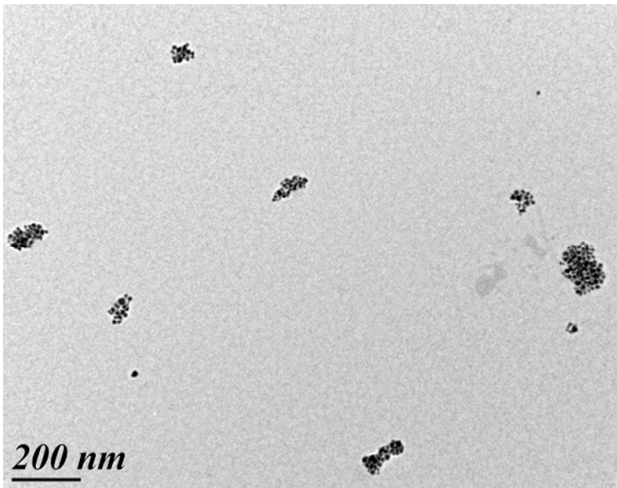

Figure SI6. TEM images showing the grapes of nanoparticles stabilized with DiC16dT (SLN ${ }^{-}$). 


\section{Synthesis of SLN ${ }_{\text {peg }}^{-}$}

Size Distribution by Intensity

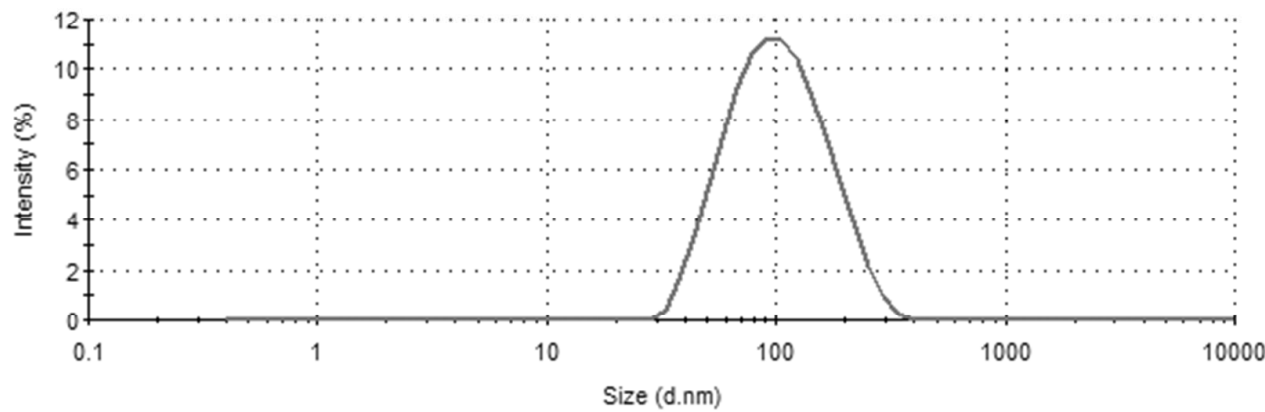

Figure SI7. Size distribution measurement of $\mathrm{SLN}_{\mathrm{peg}}^{-}$.

Zeta Potential Distribution

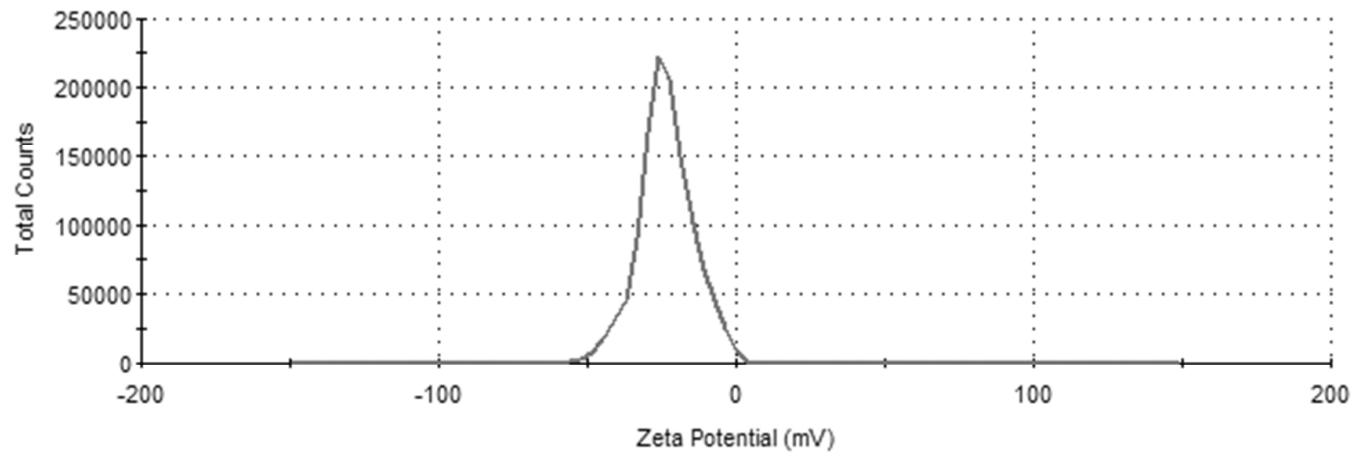

Figure SI8. Zeta potential distribution measurement of $\mathrm{SLN}_{\text {peg. }}^{-}$.

\section{Control (DOPC with iron oxide nanoparticles)}
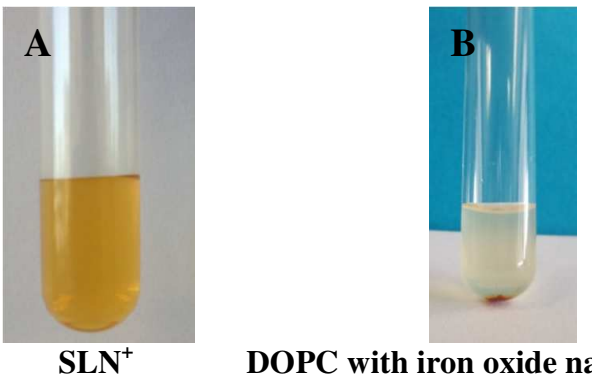

DOPC with iron oxide nanoparticles

Figure SI9. Photographs showing the solution of nanoparticles $\left(\mathrm{A}, \mathrm{SLN}^{+}\right)$and the precipitate obtained in the absence of nucleolipid (B). 


\section{B. Characterization of iron oxide nanoparticles clusters with nucleolipid and API}

\section{Synthesis of $\mathrm{SLN}^{+}$Toco}

Preparation of a lipid-based (DOTAU) nanocarrier composition containing iron oxide nanoparticles and $\alpha$-tocopherol.

$$
\text { Size Distribution by Intensity }
$$

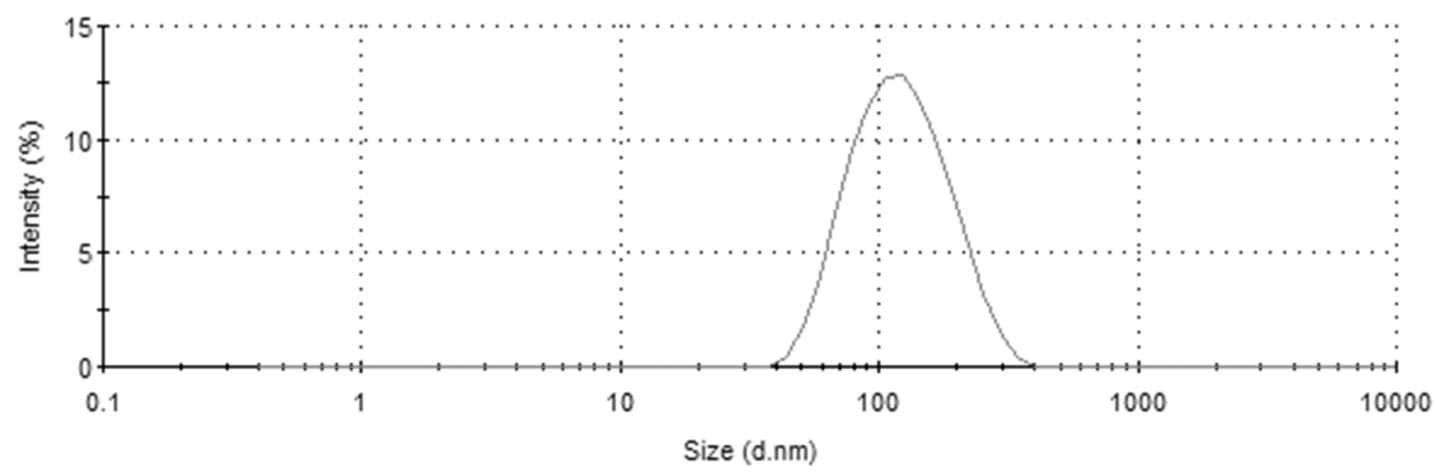

Figure SI10. Size distribution measurement of $\mathrm{SLN}^{+}$Toco.

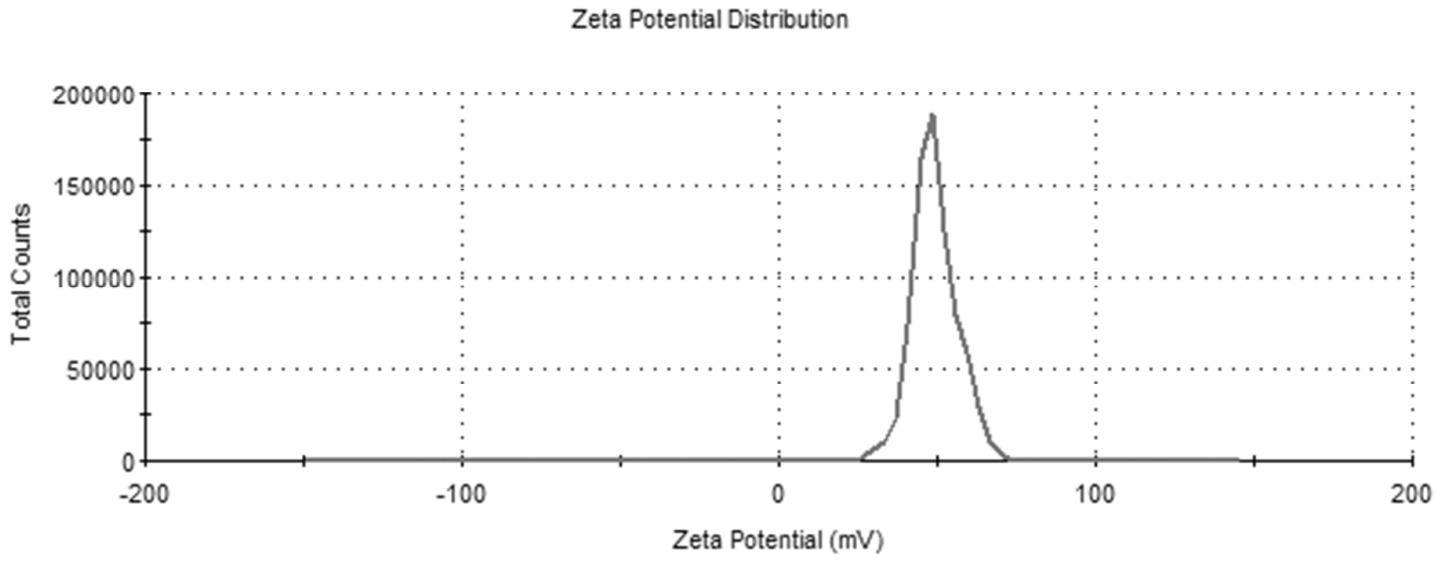

Figure SI11. Zeta potential distribution measurement of $\mathrm{SLN}^{+}$Toco.

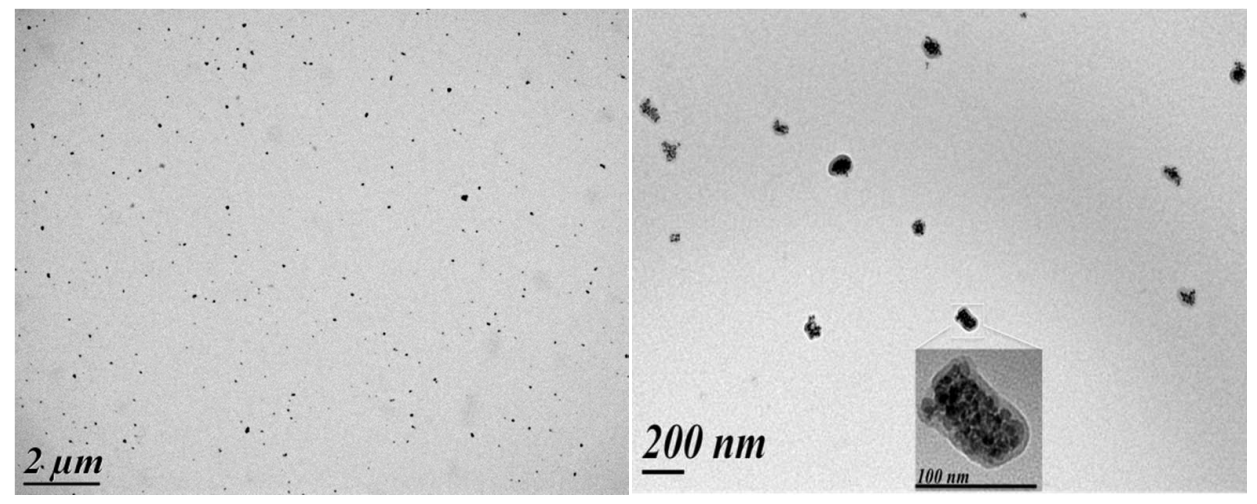

Figure SI12. TEM images showing the grapes of nanoparticles loaded with $\alpha$-tocopherol $\left(\mathrm{SLN}^{+}{ }_{\mathrm{Toco}}\right)$. 
Synthesis of SLN ${ }_{\text {Peg/PGI2 }}^{-}$

Preparation of a lipid-based (diC16dT, DOPC and DOU-PEG2000) nanocarrier composition containing iron oxide nanoparticles and prostacyclin (PGI2.Na).

Size Distribution by Intensity

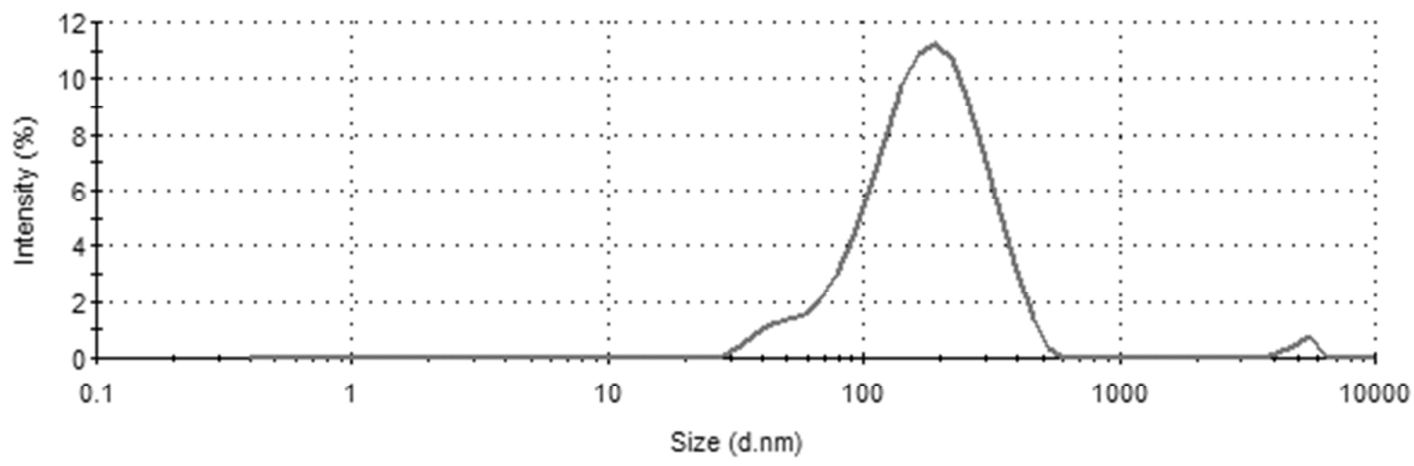

Figure SI13. Size distribution measurement of $\mathrm{SLN}^{-}{ }_{\mathrm{Peg} / \mathrm{PGI} 2}$.

Zeta Potential Distribution

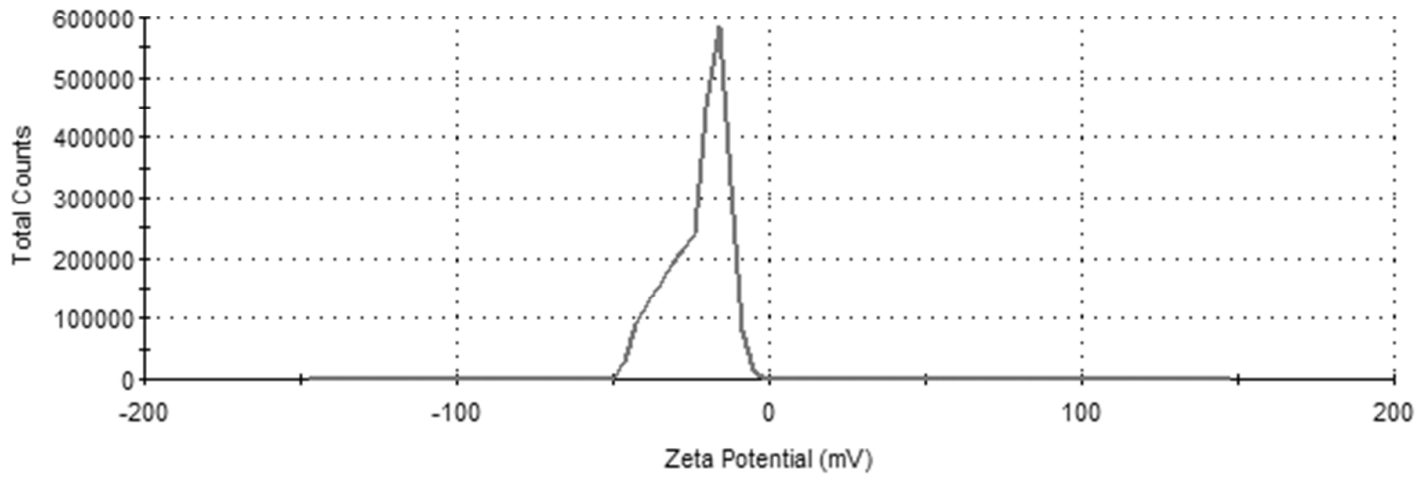

Figure SI14. Zeta potential distribution measurement of $\mathrm{SLN}_{\text {Peg/PGI2 }}^{-}$

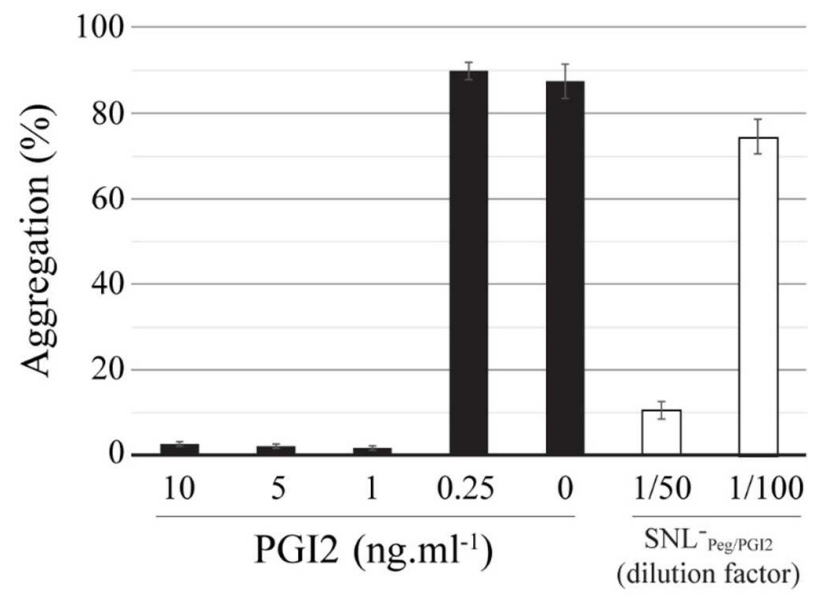

Figure SI15. Inhibition of TRAP-6 induced platelet aggregation by free PGI2 (black) and SLN ${ }_{\text {Peg/PGI2 (white) }}^{-}$ (3h of incubation). Effective PGI2 content of SLNs was estimated between 25 and $50 \mathrm{ng} / \mathrm{ml}$. 
C. Preparation of samples for HPLC analysis and dosage of DOTAU and $\alpha$-tocopherol

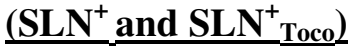

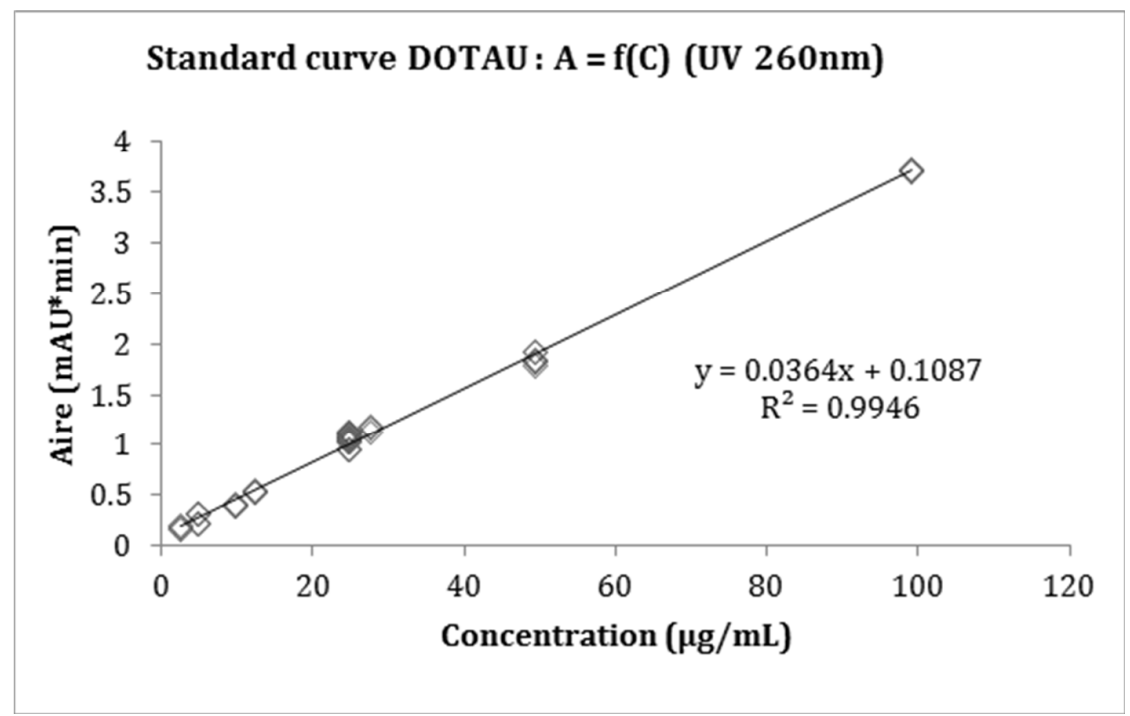

Figure SI16. Standard curve for DOTAU.

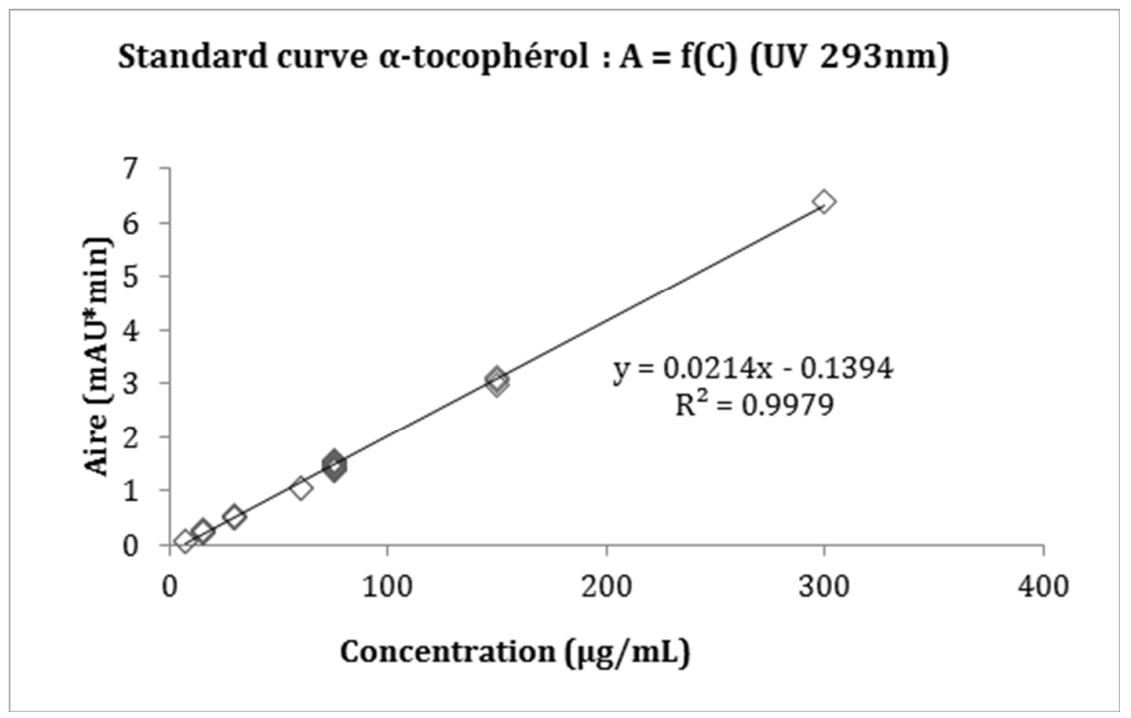

Figure SI17. Standard curve for $\alpha$-tocopherol. 

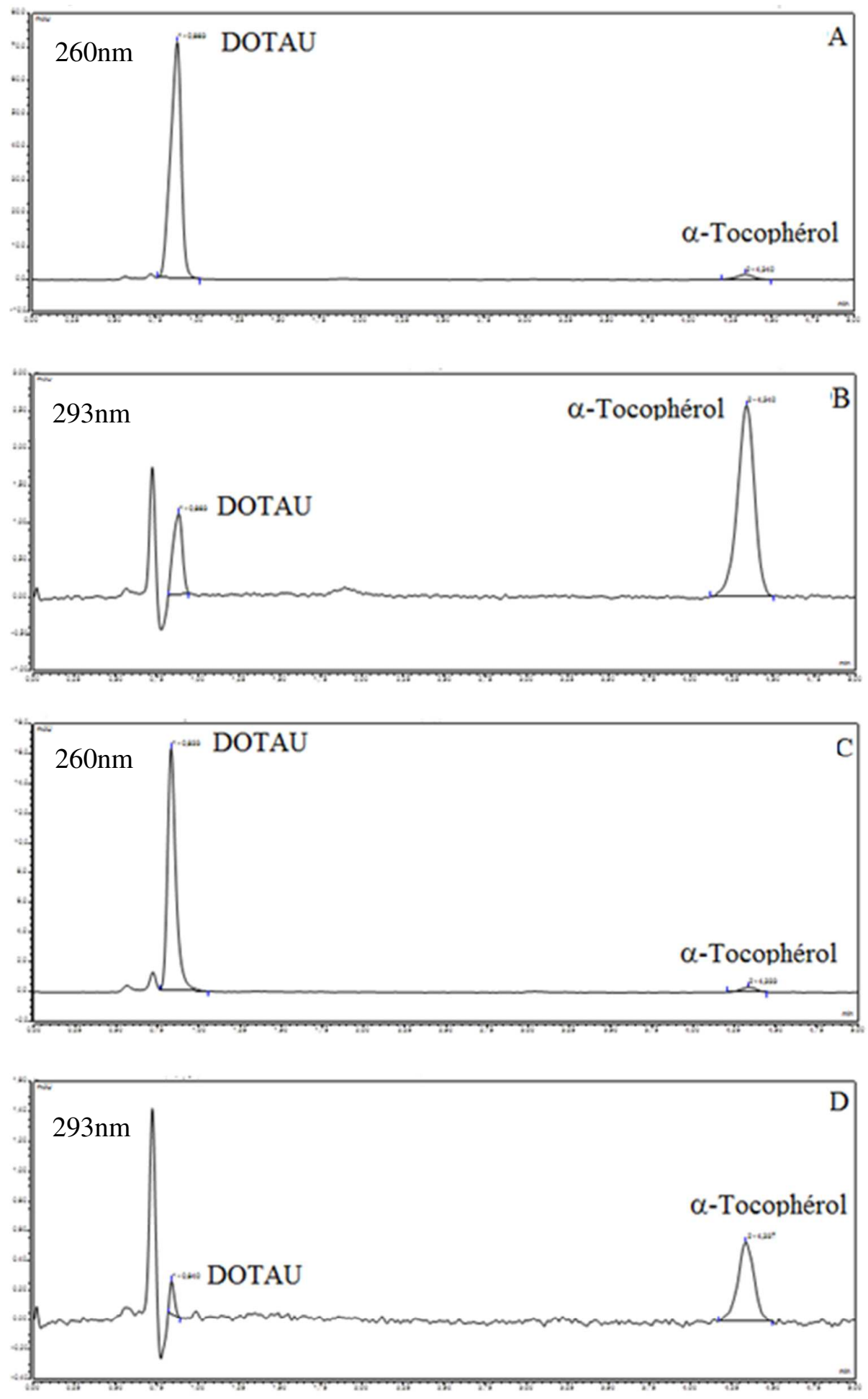

Figure SI 18. Simultaneous analysis of DOTAU and $\alpha$-Tocopherol in supernatant (A and B) and precipitate $(\mathrm{C}$ and $\mathrm{D})$. 
MR Relaxometry

\begin{tabular}{|l|l|l|l|}
\hline & r1 & r2 & $\mathbf{r}_{2}{ }^{*}$ \\
\hline SLN $^{-}$ & 1.21 & 514.6 & 556.68 \\
\hline SLN $^{+}$ & 1.26 & 279.11 & 317.3 \\
\hline Feridex® & - & - & $215[1]$ \\
\hline
\end{tabular}

Table S1.

(1) Patel, D., Kell, A., Simard, B., Xiang, B., Lin, H. Y., Tian, G. (2010) The cell labeling efficacy, cytotoxicity and relaxivity of copper-activated MRI/PET imaging contrast agents. Biomaterials, 32, 1167-1176. 